\title{
$\bullet$ IJCRR \\ Impact of COVID-19 Pandemic on Quality of Life and Medication Adherence among Pulmonary TB Patients
}

Section: Healthcare ISI Impact Factor (2019-20): 1.628 IC Value (2019): 90.81 SJIF (2020) $=7.893$

\author{
Munazzah Orooj ${ }^{1}$, Bhavna Sharma², Sumedha Rabra ${ }^{2}$, Supriya Awasthi ${ }^{3}$
}

'PhD Scholar, Department of Physiotherapy, Teerthanker Mahaveer University, Moradabad-244001, Uttar Pradesh, India; ${ }^{2}$ Assistant Professor, Department of Physiotherapy, School of Allied Health Sciences, Sharda University, Greater Noida- 201310 Uttar Pradesh, India; ${ }^{3}$ Associate Professor, Department of Physiotherapy, School of Allied Health Sciences, Sharda University, Greater Noida- 201310 Uttar Pradesh, India.

\section{ABSTRACT}

Background: The COVID-19 pandemic has presently overtaken every other health issue throughout the world. There are different ways in which current public health challenges would be impacted by this. Objective: To study on the interaction between COVID-19 and tuberculosis (TB), which still ranks as the leading cause of death from a single infectious disease globally.

Methods: A total of 250 Pulmonary Tuberculosis patients from a tertiary dot centre agreed to participate in the survey. The information regarding demographics (age and gender), medication adherence, and quality of life characteristics was collected by interviewing the participants through the questionnaires (MMAS and SGRQ) from the patients by the principal investigator before and during the pandemic. Descriptive statistics used to calculate mean, SD, and P-value.

Results: Results from the self-reported HRQOL questionnaires revealed significant impairment in overall HRQOL and domains during the lockdown. Based on the adherence grading of MMAS-8, the majority of TB patients reported a reduced medication adherence rate during the pandemic. Furthermore, there were positive associations between overall SGRQ scores and medication adherence rate during the COVID-19 pandemic as confirmed by statistically significant results.

Principle Conclusion: Our examination reasoned components such as medication adherence and QOL of patients with pulmonary tuberculosis. This study showed that more than half of patients reported poor quality of life and reduced medication adherence during the COVID-19 pandemic. A positive relationship exists between adherence and HRQOL among pulmonary TB patients.

Key Words: COVID-19 Pandemic, Medication Adherence, Pulmonary tuberculosis, Quality of Life

\section{INTRODUCTION}

The advent of major outbreaks of infectious diseases in many parts of the world has created new challenges for global and national efforts to monitor TB. ${ }^{1}$ A recent modelling study found that an additional 1.65 million TB cases and 438,000 TB deaths in India over the next 5 years will be triggered by a 3-month lockdown due to COVID-19. ${ }^{2}$ An estimated 10 million people grow active TB and more than one million people die per year from TB, according to the World Health Organization (WHO) report. ${ }^{3}$ Owing to the ongoing COVID-19 pandemic, this number would likely increase. In subsequent years, the direct and indirect effects of these viral outbreaks on TB programs have resulted in an increased burden of TB in the regions affected. The emerging COVID -19 pandemic is a global health epidemic that has caused major damage to the health care system, including the TB programs. ${ }^{4}$

One of the major preventative steps for COVID-19 is social distancing. With effect from 25 March 2020, the Government of India placed a nationwide lockdown and extended it further until 30 May 2020. ${ }^{5}$ For most countries, maintaining the smooth running of programs leading to the interruption of routine TB treatment is very difficult. There is an expectation that people with TB and COVID-19 could show unfavourable treatment results, especially in the case of coinfection.

The public health response to COVID-19 to isolate people in their homes for prolonged periods, especially in India, may promote TB transmission because close household contact is a key risk factor for TB, particularly in low socioeconomic and

\section{Corresponding Author:}

Munazza Orooj, Ph.D. Scholar, Department of Physiotherapy, Teerthanker Mahaveer University, Moradabad-244001, Uttar Pradesh India. Contact: 08750712955; Email: munazzaorooj999@gmail.com

ISSN: $2231-2196$ (Print) ISSN: 0975-5241 (Online)

Received: 22.12 .2020 Revised: 12.01 .2021 Accepted: 23.01 .2021 Published: 30.03 .2021 
overcrowded conditions. Previous studies have also shown that the prevalence of TB among children in adult households is higher than in the general population and the risk of household infection is significantly increased with prolonged household contact with sputum-positive adults. ${ }^{6-8}$

Countries with a high TB burden such as India have a significant number of patients with post-TB lung sequelae disease and the outcome of COVID-19 in these patients is unknown to date. Recent reviews have discussed the effects of COVID-19 on other diseases such as cancer and diabetes. ${ }^{9}$,

${ }_{10}$ However, no studies have been reported on the effect of COVID-19 on quality of life and adherence to medication among patients with pulmonary TB.

Therefore, we hypothesized that there will be a potential impact of COVID-19 pandemics on the health-related quality of life and its association with medication adherence among Pulmonary Tuberculosis patients in India.

\section{MATERIALS AND METHODS}

\section{Research Design and Sampling}

A Cross-sectional survey was used to collect the data from study subjects by using a validated and reliable questionnaire assessing TB therapy adherence and Quality of life among pulmonary tuberculosis patients. Patients, who were aged 18 years or above with confirmed TB diagnosis with no other co-morbidities, who can read and understood the content and questionnaire, and gave their consent to participate had included in the present survey ( Figure 1).

\section{Sample Size calculation}

We performed a priori power analysis using $G^{*}$ Power to evaluate our target sample size (version 3.1.9.2). Due to the lack of prior research to inform our expected size of effects, a previous study by Craig A. Harper et al described our effect size of interest and reported $\mathrm{r}=.20$ as the typical effect size. ${ }^{11}$ Therefore, we set a conservative error rate of form I and II both to .05 and aimed at detecting $\mathrm{r}=.20$, concluding a target sample size of $\mathrm{N}=200$. Out of that, $\mathrm{N}=250$ participants (Mean age $=37.37$ years, $\mathrm{SD}=7.71,40 \%$ female) met all four attention checks and were retained for analysis.

\section{Data Collection}

300 registered TB patients who are eligible for home care within the sample frame. Of these, 250 open TB patients who participated in the survey cases were confirmed with sputum for Acid-Fast Bacilli, Monteux, Gene expert, and Chest X-ray from August 2019 until May 2020. Before and after the lockout time, the data was collected, which is the author could not collect the data through face-to-face interviews, so we collected the data online. An introduction of the author was given to the participants, the objective of the study, the procedures for filling out the answers, the confidentiality of the participants, and the questionnaire was posted in the WhatsApp community, emails, and over the phone. Participants were asked to respond to the questionnaire in its entirety by selecting only one answer. Information on demographics (age and gender), adherence to medication, and quality of life characteristics were gathered by interviewing participants through the search.

The patients who were willing to agree to participate in the research were told of the essence and characteristics of the study and asked to give their consent. Finally, the interviewer recorded the clinical details and information about ATT through the MMAS-8 questionnaire, a general assessment and self-reported measure of drug-taking behaviour, consisting of eight items measuring drug-taking behaviour The scoring criteria can be obtained based on the overview scores from the MMAS-8 ranging from 0 to 8, and Morisky's cut-points are predetermined, adherence levels could be classified as high ( $=8$ points), medium ( 6 or 7 points) and low ( $<6$ points) Each question has two choices, i.e. yes/no. One point and an incorrect.

The zero-point answers were allocated for each correct response. ${ }^{12}$ St George's Respiratory Questionnaire (SGRQ) describes the quality of life as a disease-specific method designed to assess the effect on general health, everyday life, and perceived well-being in patients with obstructive airway disease. ${ }^{13}$ The questionnaire used St George's Respiratory Questionnaire and Morisky Medication Adherence Scale has been previously validated by Pasipodanya et al. 2003 with Cronbach alpha 0.093. ${ }^{14}$

\section{Statistical analyses}

The information collected was coded and entered into an excel sheet. Using IBM SPSS version 23, statistical analysis was performed. Descriptive research was applied to the socio-demographic baseline, Quality of life, and adherence data (N, mean, $S D$,). Spearman's correlation coefficients were calculated between HRQOL scores and adherence mean scores.

\section{RESULTS}

In the present study, there were a total of 250 participants. Of these, $60 \%$ of the surveyed patients were men, and $40 \%$ were women. The subject's demographic and clinical characteristics and scores of the MMAS-8, at baseline, are shown in Table 1. Table 2 depicts the standardized mean difference of outcome variables before and after COVID-19 lockdown between the groups with a significant difference in their pre and during lockdown values $(\mathrm{p}<0.05)$ ( Figure 2 ). 
Figure 3 and Figure 5 shows $60 \%$ of the TB patients were categorized as having low medication adherence, and $40 \%$ as having medium/high adherence Based on the adherence grading of MMAS-8, the majority of TB patients reported reduced medication adherence rate during the pandemic. Further, among the entire group, the total SGRQ score was $80 / 100$ (higher scores being worst). The readings were compared with Pre- lockdown before the COVID-19 pandemic where the SGRQ score was 12/100 (lower scores being better). Results from the self-reported HRQOL questionnaires revealed significant impairment in overall HRQOL and domains during the lockdown as shown in figure 2 . The overall changes in HRQOL were statistically significant and exceeded the published thresholds for minimal important difference (MID) indicating the differences can be interpreted as clinically meaningful.

Figure 4 presents the positive associations between overall SGRQ scores and medication adherence rate during the COVID-19 pandemic was confirmed by statistically significant results from Spearman correlation as shown in Table 3.

\section{DISCUSSION}

The outcome of this study indicates that adherence to TB care was poor and remained low during the pandemic, while treatment difficulty was a predictive factor for low adherence in other conditions. Approximately one-third of the patients interviewed were non-adherent to the treatment of TB and/or had insufficient awareness and level of commitment to TB. Several factors related to health care, treatment and patients have also been linked substantially with TB awareness and anti-TT adherence. These associations have been identified previously in other countries, ${ }^{15}$ but this is, to our knowledge, the first study carried out on this subject in India.

The SGRQ scores were higher in the present study population that significantly leads to impairment in their quality of life. The scores obtained from the study population were compared with mean values of the same population before lockdown and also with the mean normal values of the general population as per the recommendations in the SGRQ manual. ${ }^{13}$ The symptoms, activity, and impact mean scores in the studied population during lockdown were 80, 70, 68 which was high when compared to their Pre lockdown scores of $35,25,12$, as well as general population scores of 12,9 and 2. These findings were similar previous study. ${ }^{14}$

One of the steps that countries have taken to prevent COVID-19 from spreading is to require people to remain at home before the situation becomes normal. ${ }^{15}$ We also observed that poorer quality of life was associated significantly with lower adherence to TB medications. Another research conducted in Brazil found that household exposure severity increased household members' risk of TB infection and disease. ${ }^{16}$ Family support was previously identified as having a strong influence on patient adherence to TB care in low-income countries, including financial assistance, self-motivation, and emotional support. ${ }^{17}$

A study conducted by Rossi et al shows relatively high rates of symptoms of depression, symptoms of anxiety, insomnia with a variety of risk factors associated with COVID, including being under quarantine, having a loved one deceased by COVID-19, stopping work due to lockdown steps, or witnessing other stressful events (i.e. working, financial, relationship or housing problems) due to the pandemic or housing problems..$^{18}$ In many patients with chronic conditions, depression persists as a comorbid disorder. Depression was shown to be substantially linked to a low degree of adherence to medication in TB patients, and the fact that depression increased the risk of medication non-adherence has been found in many other studies. ${ }^{19}$ A meta-analysis found that the risk of non-adherence in depressed patients was three times higher than in non-depressed patients. ${ }^{20}$

It was the first time that MMAS- 8 was applied to an adherence study in pulmonary TB patients in India to provide a simple and inexpensive measuring instrument of primary importance for many adherence studies to our knowledge. The scale showed that more than $(60 \%)$ of TB patients had poor adherence to medication, which is a condition that cannot be overlooked when trying to control TB. Theron et al. measured psychological distress in TB patients in southern Africa and found a strong association between psychological distress and non-adherence correlated with higher levels of $\mathrm{TB}^{21}$

In terms of higher levels of anxiety and depression, there was a positive correlation between adherence and HRQOL during the lockdown and the overall HRQOL result of our research is in line with other studies that recorded that adherence in patients with COPD was positively affected by impaired HRQOL. ${ }^{22}$ This study also has many benefits, including a very broad sample size and the sampling period.

\section{CONCLUSION}

Tuberculosis is only one of the regions that would be sidelined and adversely impacted by the Covid-19 Pandemic in global pandemic health. More than half of the patients reported poor quality of life and medication adherence, this study showed. The COVID-19 pandemic has affected TB control in a variety of ways, including increased household TB transmission, delay, diagnosis, and TB care. 


\section{FUTURE RECOMMENDATIONS}

There is no doubt that India is making every effort to stop the COVID-19 pandemic, but it is not appropriate to ignore the old silent killer TB that is preventable and treatable. Focusing solely on COVID-19 will result in harm to the fragile gains made under the WHO end TB strategy for the elimination of TB. Strategies implemented to curb both TB and COVID-19 epidemics will supplement each other, contributing to an overall mortality reduction. This will involve our government, the public and the private health sector to take a concerted approach. India will also benefit from realising the dream of ending TB by 2025 if these resources can be used in the TB elimination program.

\section{ACKNOWLEDGEMENT}

The authors thank all the participants for their cooperation in completing the study. The authors are also grateful to authors/editors/publishers of all those articles, journals, and books from where the literature for this article has been reviewed and discussed. We are extremely thankful to all the contributors who are actively participated to complete the study in this pandemic.

\section{Conflict of Interest: None declared}

Funding Statement: No fund received for this study

\section{REFERENCES}

1. Alene KA, Wangdi K, Clements AC. Impact of the COVID-19 pandemic on tuberculosis control: an overview. Trop Med Infect Dis 2020;5(3):123.

2. Cilloni L, Fu H, Vesga JF, Dowdy D, Pretorius C, Ahmedov S, et al. The potential impact of the COVID-19 pandemic on tuberculosis: a modelling analysis. 2020. medRxiv preprint doi: https:// doi.org/10.1101/2020.05.16.20104075

3. World Health Organization. Global status report on alcohol and health 2018.

4. Hogan AB, Jewell BL, Sherrard-Smith E, Vesga JF, Watson OJ, Whittaker C, et al. Potential impact of the COVID-19 pandemic on HIV, tuberculosis, and malaria in low-income and middleincome countries: a modelling study. Lancet Glob Health 2020;8(9):e1132-41.

5. Ministry of Health and Family Welfare, Government of India. GOI RSS, New Delhi, India. Available from http;/www.mohfw. gov.in/ [Accessed 2020 June 20]

6. Singh M, Mynak ML, Kumar L, Mathew JL, Jindal SK. Prevalence and risk factors for transmission of infection among children in household contact with adults having pulmonary tuberculosis. Arch Dis Childhood 2005;90(6):624-628.

7. Lalor MK, Anderson LF, Hamblion EL, Burkitt A, Davidson JA, Maguire H, Abubakar I, Thomas HL. Recent household trans- mission of tuberculosis in England, 2010-2012: retrospective national cohort study combining epidemiological and molecular strain typing data. BMC Med 2017;15(1):105.

8. Saunders MJ, Wingfield T, Datta S, Montoya R, Ramos E, Baldwin MR, et al. A household-level score to predict the risk of tuberculosis among contacts of patients with tuberculosis: a derivation and external validation prospective cohort study. Lancet Infect Dis 2020;20(1):110-122.

9. Hanna TP, Evans GA, Booth CM. Cancer, COVID-19 and the precautionary principle: prioritizing treatment during a global pandemic. Nature Rev Clin Oncol 2020;17(5):268-270.

10. Maddaloni E, Buzzetti R. Covid-19 and diabetes mellitus: unveiling the interaction of two pandemics. Diab Metab Res Rev 2020:e33213321.

11. Harper CA, Satchell LP, Fido D, Latzman RD. Functional fear predicts public health compliance in the COVID-19 pandemic. Int J Ment Health Addict 2020 Apr 27:1-14.

12. Xu M, Markström U, Lyu J, Xu L. Detection of low adherence in rural tuberculosis patients in China: application of Morisky medication adherence scale. Int J Environ Res Public Health 2017;14(3):248.

13. Jones PW. St. George's respiratory questionnaire: MCID. J Chronic Obstr Pulmon Dis 2005;2(1):75-79.

14. Pasipanodya JG, Miller TL, Vecino M, Munguia G, Bae S, Drewyer G, Weis SE. Using the St. George respiratory questionnaire to ascertain health quality in persons with treated pulmonary tuberculosis. Chest 2007;132(5):1591-1598.

15. Kastien-Hilka T, Rosenkranz B, Schwenkglenks M, Bennett BM, Sinanovic E. Association between health-related quality of life and medication adherence in pulmonary tuberculosis in South Africa. Front Pharmacol 2017;8:919.

16. Anderson RM, Heesterbeek H, Klinkenberg D, Hollingsworth TD. How will country-based mitigation measures influence the course of the COVID-19 epidemic? Lancet 2020;395(10228):931-934.

17. Harper M, Ahmadu FA, Ogden JA, McAdam KP, Lienhardt C. Identifying the determinants of tuberculosis control in resourcepoor countries: insights from a qualitative study in The Gambia. Transact Royal Soc Trop Med Hygi 2003;97(5):506-510.

18. Rossi R, Socci V, Talevi D, Mensi S, Niolu C, Pacitti F, Di Marco A, Rossi A, Siracusano A, Di Lorenzo G. COVID-19 pandemic and lockdown measures impact on mental health among the general population in Italy. Front Psychia 2020;11:790.

19. Tang Y, Zhao M, Wang Y, Gong Y, Yin X, Zhao A, et al. Non-adherence to anti-tuberculosis treatment among internal migrants with pulmonary tuberculosis in Shenzhen, China: a cross-sectional study. BMC Public Health 2015;15(1):474.

20. DiMatteo MR, Lepper HS, Croghan TW. Depression is a risk factor for noncompliance with medical treatment: meta-analysis of the effects of anxiety and depression on patient adherence. Arch Inter Med 2000;160(14):2101-2107

21. Theron G, Peter J, Zijenah L, Chanda D, Mangu C, Clowes P, et al. Psychological distress and its relationship with non-adherence to TB treatment: a multicentre study. BMC Infect Dis 2015;15(1):253.

22. Ágh T, Dömötör P, Bártfai Z, Inotai A, Fujsz E, Mészáros Á. Relationship between medication adherence and health-related quality of life in subjects with COPD: a systematic review. Resp Care 2015;60(2):297-303. 
Table 1: Subject's demographic and clinical characteristics at baseline $(n=250)$

\begin{tabular}{lccc} 
Variable & Mean & SD & $p$-value \\
Age, years & 37.37 & 17.516 & 0.37 \\
Height, cm & 160.65 & 14.604 & 0.94 \\
Weight, kg & 57.81 & 10.322 & 0.54 \\
BMI, kg/cm ${ }^{2}$ & 22.47 & 3.917 & 0.50 \\
MMRC & 12.24 & 3.70 & 0.02 \\
Treatment length & & & \\
< 3 months & 59.92 & 11.425 & 0.80 \\
>3months & 68.24 & 12.872 & 0.03 \\
$\quad$ SGRQ(HRQOL) & & & \\
SGRQ SYMP & 34.524 & 5.642 & 0.41 \\
SGRQ IMPACT & 18.064 & 3.912 & 0.42 \\
SGRQACTIVITY & 34.592 & 5.487 & 0.43 \\
SGRQ TOTAL & 31.232 & 19.902 & 0.67 \\
MMAS (Medication & 32.28 & 8.232 & 0.71 \\
Adhereance) & & & \\
\hline
\end{tabular}

Values are expressed as means, standard deviation unless otherwise stated. Significance level $p<0.050$, BMI body mass index, mMrc modified medical research council, SGRQ St Georgee Respiratory Questionnaire, MMAS Morisky Medication Adherence Scale

Table 2: Standardized mean difference of outcome variables after before and After COVID-19 lockdown between the groups

\begin{tabular}{llccc}
$\begin{array}{l}\text { Within } \\
\text { Group }\end{array}$ & Before-After & Mean difference & SD & P Value \\
Pair1 & Borg & 1.120 & 1.3741 & 0.00o \\
Pair2 & SGRQ Symp & 33.108 & 16.831 & 0.000 \\
Pair3 & SGRQ Impact & 22.316 & 9.210 & 0.000 \\
Pair4 & SGRQ activity & 38.604 & 8.424 & 0.000 \\
Pair5 & SGRQ total & 43.756 & 22.261 & 0.000 \\
Pair6 & MMAS & 8.324 & 0.837 & 0.00o \\
\hline
\end{tabular}

Values are expressed as means, standard deviation unless otherwise stated and significance level $\mathrm{p}<0.050$. SGRQ St Georgee Respiratory Questionnaire, MMAS Morisky Medication Adherence Scale

Table 3: Correlation between Medication adherence and Quality of life among pulmonary TB patients during COVID-19

$\begin{array}{ccc}\text { MMAS } & \text { r value } & \text { P value } \\ \text { ITEM1 } & 0.55 & 0.01 \\ \text { ITEM 2 } & 0.45 & 0.01 \\ \text { ITEM }_{3} & 0.64 & 0.01\end{array}$

Table 3: (Continued)

\begin{tabular}{lccc} 
& MMAS & r value & P value \\
$\begin{array}{l}\text { Quality of life (Total SGRQ } \\
\text { Score) }\end{array}$ & ITEM 4 & 0.51 & 0.02 \\
& & & \\
& ITEM5 & 0.53 & 0.04 \\
& ITEM6 & 0.57 & 0.05 \\
& ITEM 7 & 0.48 & 0.03 \\
& ITEM 8 & 0.83 & 0.03 \\
\hline
\end{tabular}

Values are expressed as $r$ correlation coefficient and significance level $p<0.050$. SGRQ St Georgee Respiratory Questionnaire, MMAS Morisky Medication Adherence Scale

\section{Pulmonary Tuberculosis patients aged $18-60$ were included}

Informed consent was taken

SGRQ and MMAS Questionnaire was taken from participant

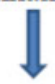

Quality of life and Medication adherence rate before and during COVID-19 Lockdown were recorded

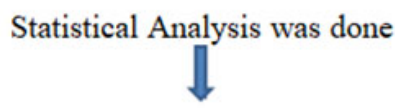

Result

Figure 1: Study flow.

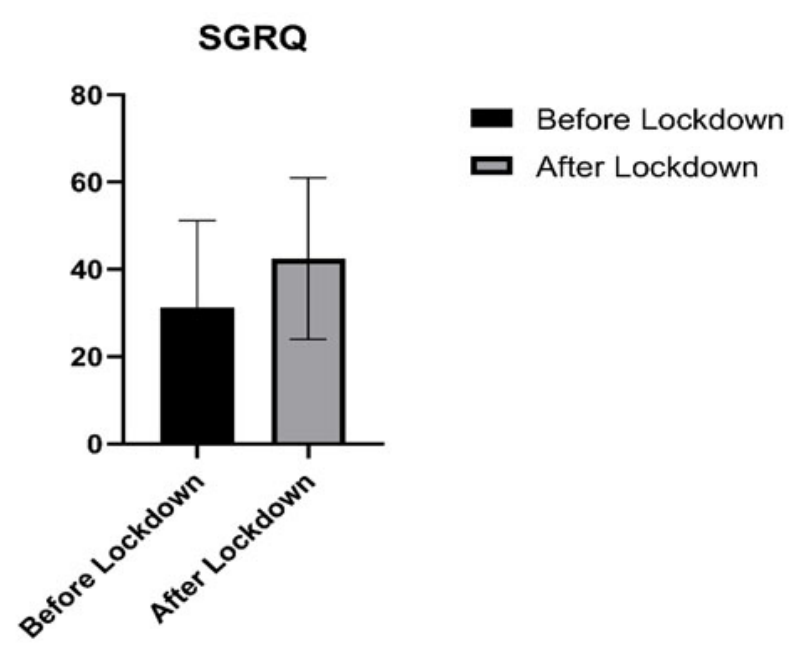

Figure 2: Pre-Post SGRQ Scores. 

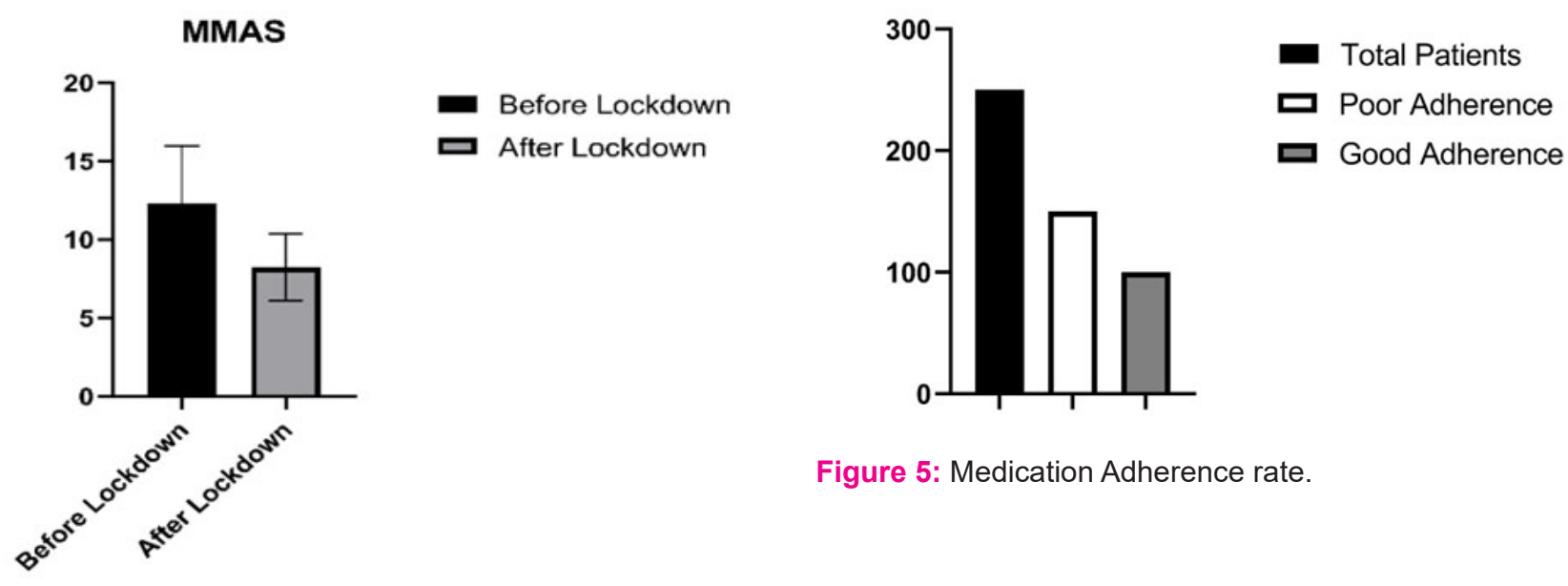

Figure 5: Medication Adherence rate.

Figure 3: Pre-Post MMAS Scores.

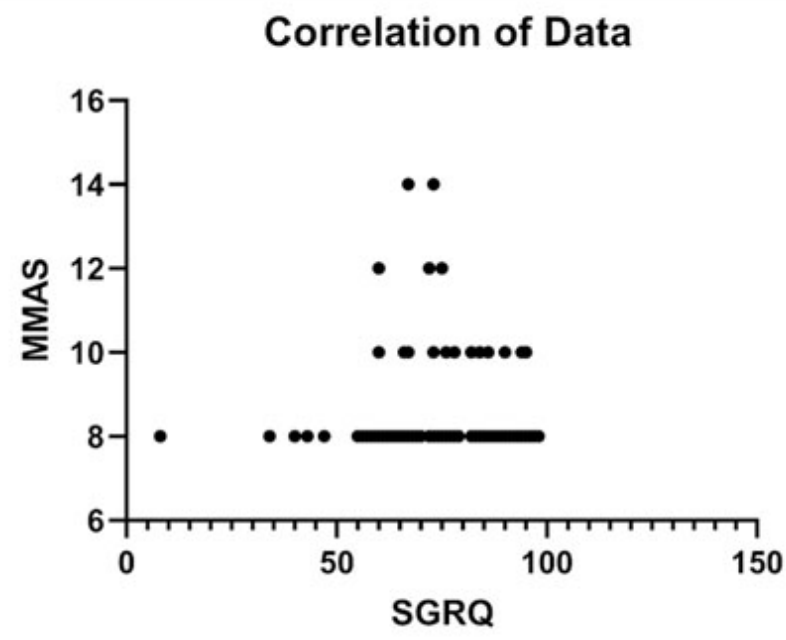

Figure 4: Correlation between SGRQ and Mmas. 